\title{
Sachwortverzeichnis/Subject Index 1991
}

A

Adipositas, 47

Agoraphobie, 26, 66

Alkoholabhängigkeit, 301

Ambulante psychothera-peutische Versorgung, 219

Ambulante Verhaltenstherapie, 15

Angst, 6

Angstanfälle, 34

Angststörungen, 34, 55, 273

Anorexia nervosa, 47, 61

Anti-Diät-Konzept, 47

Atemanhalten, 34

B

Bewältigungsstrategien,

212 Blutdruck, 120 Bulimia nervosa, 47,61

C

Coping, 130

D

Delegationsverfahren, 15

Depression, 6, 139, 200,

207 Depressives Interaktions-

verhalten, 207 Diagnostik, 110 DSM-III-R, 110

E

Exposition in vivo bei Pa-

thologischem Spielen,

307 Expressed Emotion,

283

$\mathrm{F}$

Familiäre Interaktion,

283 Formale Denkstörungen,

200

G

Gesundheitspolitik, 15,

219 Grenzwerthypertonie,

120

$\mathrm{H}$

Hamburger Zwangs-Inventar (HZI), 223

Hyperventilation, 34 Hypotonie, 120

$\mathrm{K}$ 
Kognitive Therapie, 6,

200 Komorbidität, 273 Krankheitsbewältigung,

293

M Mimik, 120

$\mathrm{N}$

Nonverbale Kommunika-tion, 293

$\mathrm{P}$

Panikattacken, 26 Panikstörung, 66 Pathologisches Spielen,

307 Phobie, 55 Plazebo, 26 Prävalenz, 273 Psychische Störungen,

110 Psychoedukative Fami-

lientherapie, 283

Psychopharmakologische

Therapie, 26 Psychotherapie, empiri-

sche Ergebnisse, Über-

sichtsartikel, 130 Psychotherapie-Kosten,

15 Psychotherapie-Richt-

linien, 15

$\mathrm{S}$

Schizophrene Patienten,

212 Schizophrenie, 130, 283,

293 Selbsthilfe, 55 Selbstkontrolle, 307 Sexualstörung, 139 Soziale Ängste, 34 Soziale

Interaktion, 207 Spontanremission, 273 Spontanverlauf, 273 Stationäre Psychotherapie, 219

Stationäre verhaltensme-dizinische Behandlung, 47 Stationäre Verhaltenstherapie, 307

Streß, 120,130, 212 Strukturierte Interviews,

110,297 Suchterkrankungen,

301

$\mathrm{T}$

Tagklinische Behandlung, 61

Tests zur Zwangssympto-matik, 223

Therapie-Manual, 66

Therapieplanung, 110

$\mathrm{V}$

Verbale Kommunikation,

283 Verbalverhalten, 207 Verhaltensmedizin, 219 Verhaltenstherapie, 66,

301

$\mathrm{Y}$

Yale-Brown Obsessive Compulsive Scale (Y-BOCS), 223

$\mathrm{Z}$

Zwangskrankheit, 223 